\title{
On the MHD Boundary of Kelvin-Helmholtz Stability Diagram at Large Wavelengths
}

\author{
F. T. Gratton ${ }^{1}$, G. Gnavi ${ }^{1}$, C. J. Farrugia ${ }^{2}$, and L. Bender ${ }^{1}$ \\ ${ }^{1}$ Instituto de Física del Plasma, Facultad de Ciencias Exactas y Naturales, \\ Universidad de Buenos Aires y Consejo Nacional de Investigaciones Científicas y Técnicas, \\ Ciudad Universitaria, 1428 Buenos Aires, Argentina \\ ${ }^{2}$ Space Science Center, University of New Hampshire, Durham, NH, 03824, USA
}

Received on 08 February, 2004; revised version received on 14 May, 2004

\begin{abstract}
Working within the domain of inviscid incompressible MHD theory, we found that a tangential discontinuity (TD) separating two uniform regions of different density, velocity and magnetic field may be Kelvin-Helmholtz $(\mathrm{KH})$ stable and yet a study of a transition between the same constant regions given by a continuous velocity profile shows the presence of the instability with significant growth rates. Since the cause of the instability stems from the velocity gradient, and since a TD may be considered as the ultimate limit of such gradient, the statement comes as a surprise. In fact, a long wavelength $(\lambda)$ boundary for the $\mathrm{KH}$ instability does not exist in ordinary liquids being instead a consequence of the presence of magnetic shear, a possibility that has passed unnoticed in the literature. It is shown that $\mathrm{KH}$ modes of a magnetic field configuration with constant direction do not have the long $\lambda$ boundary. A theoretical explanation of this feature and examples of the violation of the TD stability condition are given using a model that can be solved in closed form. Stability diagrams in the $(k d$, $M_{A}$ ) plane are given (where $k d=2 \pi d / \lambda, 2 d$ is the velocity gradient length scale, and $M_{A}$ is the Alfvénic Mach number) that show both the well-known limit at small $\lambda \mathrm{s}$ and the boundary for large but finite $\lambda$ s noted here. Consequences of this issue are relevant for stability studies of the dayside magnetopause as the stability condition for a TD should be used with care in data analysis work.
\end{abstract}

\section{Introduction}

The importance of the Kelvin-Helmholtz (KH) instability of parallel flows in laboratory, geophysical, or astrophysical systems, recognized many years ago, has generated a huge literature. An internet search covering only the year 2003 yielded over 280 entries. Theoretical works on the KH instability are numerous. For overviews, among many others see Chandrasekhar [1], Drazin and Reid [2], Belmont and Chanteur [3], Huerre and Rossi [4], Farrugia, Gratton and Torbert [5], and the references quoted in these surveys.

Working within the domain of inviscid incompressible MHD theory, we find that a tangential discontinuity between two uniform regions of different density, velocity and magnetic field may be Kelvin-Helmholtz stable, and yet a smooth transition (modeled with continuous profiles) between the same constant regions shows the existence of unstable modes (with equal orientation of the $\vec{k}$-vector) with significant growth rates. Since the cause of the instability stems from the velocity gradient, and since a tangential discontinuity (TD) may be considered as the ultimate limit of such gradient, the statement may be surprising at first sight.

The assumption that a TD is the worst case from the point of view of stability may lead to the erroneous inference that to decide on the stability of a particular parallel flow it is sufficient to examine the criterion of the related TD (also called the thin model). The tangential discontinuity is a simple planar interface across which the unperturbed fields, velocity $\overrightarrow{V_{0}}$, magnetic strength, $\overrightarrow{B_{0}}$, and density $\rho_{0}$, suffer sudden changes. According to incompressible MHD theory, a TD is Kelvin-Helmholtz stable when the following relation is satisfied:

$$
\frac{\rho_{0,1} \rho_{0,2}}{\rho_{0,1}+\rho_{0,2}}\left(V_{\kappa, 1}-V_{\kappa, 2}\right)^{2}<\frac{1}{4 \pi}\left[\left(B_{\kappa, 1}\right)^{2}+\left(B_{\kappa, 2}\right)^{2}\right] .
$$

Here the indices 1 and 2 refer to quantities on either side of the interface, and $V_{\kappa}, B_{\kappa}$ are the projections of $\overrightarrow{V_{0}}$ and $\overrightarrow{B_{0}}$ on the direction of the wave vector $\vec{k}$, respectively. The criterion depends on the direction of $\vec{k}$ but not on its absolute value, $k=|\vec{k}|$, and hence it is valid for all wavelengths, $\lambda=2 \pi / k$ associated to modes with a fixed $\vec{k}$ orientation.

The assumption that the stability of a parallel flow is ensured by the stability criterium of the associated TD does not hold in general for MHD. In a magnetohydrodynamic flow, and for a fixed orientation of the $\vec{k}$-vector, besides the well-known short wavelength stability boundary $\lambda_{s}$ (such that modes with $\lambda \leq \lambda_{s}$ are stable) there are configurations in which also a long wavelength limit $\lambda_{l}$ exists (such that all modes with $\lambda \geq \lambda_{l}$ are KH stable). In other words, the unstable $\lambda$ interval may be finite, $\lambda_{s} \leq \lambda \leq \lambda_{l}$, instead 
of semi-infinite, $\lambda_{s} \leq \lambda<\infty$, as it is widely assumed.

The long wavelength $(\lambda)$ stability boundary is a consequence of the presence of magnetic shear in MHD flows, a possibility that seems to have been passed unnoticed in the literature. Indeed, a long $\lambda$ boundary for the $\mathrm{KH}$ instability does not exist in ordinary non conducting fluids, where there is no process equivalent to the magnetic field stabilization. The surface tension operates only at the interface of two liquids, and it is not similar to the action of a magnetic field, the latter being an effect operating in the whole volume of the flow.

In this paper we give examples and a theoretical explanation of the violation of the stability condition 1 at moderate $\lambda \mathrm{s}$, using a model that can be solved in closed form in which the fields have idealized profiles. We present stability diagrams in the $\left(k d, M_{A}\right)$ plane (where $k d=2 \pi d / \lambda, 2 d=\Delta$ is the scale length of the velocity gradient, and $M_{A}$ is the Alfvénic Mach number) which shows both the well-known limit at small $\lambda \mathrm{s}$ and the new boundary for large, but finite $\lambda$ values. We also show, by solving numerically the stability problem of a similar model built with smooth (hyperbolic tangent) profiles, that a MHD flow with a uniformly oriented magnetic field does not have the long $\lambda$ stability boundary.

Among the possible applications of this feature of the KH instability perhaps one of the most important is found in space physics, related to the data analysis of spacecraft crossings of the terrestrial magnetopause A study of the $\mathrm{KH}$ instability of configurations that become stable at large $\lambda \mathrm{s}$ due to the presence of magnetic shear, relevant to magnetospheric physics has been published elsewhere Gratton et al. [6]. Here we present a different set of theoretical results of general nature, not aimed at a particular application.

\section{The basic equation of the MHD Kelvin-Helmholtz instability for in- compressible flows}

Our study of the Kelvin-Helmholtz instability is based on the perturbative linear equation derived from the set of ideal MHD equations (see, e.g., reference [1])

$$
\begin{aligned}
\rho \frac{d \vec{v}}{d t} & =-\operatorname{grad}(p)+\frac{1}{4 \pi} \operatorname{rot}(\vec{B}) \times \vec{B}, \quad \operatorname{div}(\vec{B})=0 \\
\frac{d}{d t}\left(\frac{\vec{B}}{\rho}\right) & =\frac{\vec{B}}{\rho} \cdot \operatorname{grad}(\vec{v}), \quad \frac{d \rho}{d t}=-\operatorname{\rho div}(\vec{v}) .
\end{aligned}
$$

Given a set of Cartesian axes, $(x, y, z)$, such that the main stream is lying on $(x, z)$ planes, all equilibrium fields $\vec{V}_{0}, \vec{B}_{0}, \rho_{0}$ are stratified across the flow and depend on $y$ only,

$$
\begin{aligned}
\vec{v} & =\left(V_{0 x}(y)+v_{x}^{1}, v_{y}^{1}, V_{0 z}(y)+v_{z}^{1}\right), \\
\vec{B} & =\left(B_{0 x}(y)+B_{x}^{1}, v_{y}^{1}, B_{0 z}(y)+B_{z}^{1}\right), \\
\rho & =\rho_{0}(y)+\rho^{1} .
\end{aligned}
$$

The $y$ component of the amplitude of a Lagrangian displacement of an infinitesimal plasma element is denoted by $\widetilde{\zeta}=\widetilde{\zeta}(x, y, z, t)$. The displacement $\widetilde{\zeta}$ is related to the $y$ component of the perturbative velocity by $v_{y}^{1}=d \widetilde{\zeta} / d t$. The dependence of the modes is assumed to be of the form

$$
\widetilde{\zeta}=\zeta(y) \exp \left(-i \omega t+i k_{x} x+i k_{z} z\right) .
$$

The real wavevector $\vec{k}=\left(k_{x}, 0, k_{z}\right)$ lies on the $(x, z)$ plane, the coordinate $y$ is in the direction transversal to the motion, and $k=\sqrt{k_{x}^{2}+k_{z}^{2}}$, the absolute value of $\vec{k}$, defines the wavelength of the mode.

A derivation of the equation that governs the mode amplitude $\zeta(y)$ (omitted here for brevity) can be found, for instance, in Farrugia et al. [7]. The equation is

$$
\frac{d}{d y}\left(\mathcal{H} \frac{d \zeta}{d y}\right)-k^{2} \mathcal{H} \zeta=0
$$

and is obtained under the condition $\operatorname{div}\left(\overrightarrow{v^{1}}\right)=0$ that corresponds to the incompressible approximation. Given $\vec{k}$, the characteristic boundary value problem for equation 5 determines both the complex frequency $\omega$ and the mode eigenfunction $\zeta(y)$. The imaginary part of $\omega, \gamma=\Im(\omega)$, gives the growth rate of the instability (when $\gamma>0$ ) and the real part, $\Re(\omega)$, gives the frequency of oscillation of the wave.

The function $\mathcal{H}=\mathcal{H}(y ; \vec{k}, \omega)$ in eq.5 is defined by

$$
\mathcal{H} \equiv \rho_{0}(y)\left[\left(c-V_{\kappa}(y)\right)^{2}-V_{A \kappa}^{2}(y)\right]
$$

where $c$ is the (complex) phase velocity

$$
c=\frac{\omega}{k} \text {. }
$$

In eq.6, as already mentioned in the introduction, $\rho_{0}=$ $\rho_{0}(y)$ represents the equilibrium density across the flow, $V_{\kappa}(y)=\overrightarrow{V_{0}} \cdot \vec{\kappa}$ is the projection of the equilibrium velocity $\overrightarrow{V_{0}}=\overrightarrow{V_{0}}(y)$ on the wavevector direction $\vec{\kappa}=\vec{k} / k$, while $B_{\kappa}=B_{\kappa}(y)=\overrightarrow{B_{0}} \cdot \vec{\kappa}$ is the projection of the equilibrium magnetic field. Finally, we have introduced the projection of the Alfvén velocity on $\vec{\kappa}, V_{A \kappa}$, defined by

$$
V_{A \kappa}^{2}(y)=\frac{B_{\kappa}^{2}(y)}{4 \pi \rho_{0}(y)} .
$$


The reader must note that equation 5 is valid for modes with all $\lambda \mathrm{s}$, but with a fixed $\vec{\kappa}$. A change of the wavevector direction leads to a different set of field projections, $V_{\kappa}(y)$, $B_{\kappa}(y)$ and so to a different eq. 5 . Thus, a complete stability analysis of a given parallel flow, requires not only a study of all $\lambda \mathrm{s}$ modes, but also an examination of all the orientations of $\vec{\kappa}$ on the $(x, z)$ plane. In the following we ordinarily consider only fixed $\vec{\kappa}$ modes, and therefore the analysis is restricted to a particular direction of the wavevector.

The theory based on eq.5 is restricted to incompressible perturbations. It can be shown (e.g., Contin et al. [8], Gratton et al. [9]) that the relative change of density due to plasma advection, $\left(\delta \rho / \rho_{0}\right)_{a}$, for $\mathrm{KH}$ modes, is much larger than the variation due to compressibility, $\left(\delta \rho / \rho_{0}\right)_{c}$, when the velocity $U=\left|\overrightarrow{V_{0}}\right|$ is much smaller than the local speed of sound, $C_{s}$. In fact, it turns out that one can estimate the ratio of density changes caused by the two mentioned processes as

$$
\frac{\left(\delta \rho / \rho_{0}\right)_{c}}{\left(\delta \rho / \rho_{0}\right)_{a}} \sim\left(\frac{U}{C_{s}}\right)^{2}
$$

Thus, compressibility is a minor correction in the perturbative analysis of subsonic flows when

$$
\left(\frac{U}{C_{s}}\right)^{2} \ll 1
$$

We may note that in $y$ intervals where $\mathcal{H}$ is constant there are two simple exponential solutions $\exp ( \pm k y)$ of eq.5. These solutions represent the KH perturbation on both sides of a layer that separates two regions of constant field with different values. When the wavelength $\lambda$ becomes much larger than the thickness $\Delta$ of the interface, the field profiles $\rho_{0}, B_{\kappa}$, or $V_{\kappa}$ can be approximated by step functions with a discontinuity (TD) at the position $y=y_{0}$ where the transition occurs. Thus, when $\lambda \gg \Delta$, the $\mathrm{KH}$ modes do not contain any information on the structure of the transition, and are forced to be piecewise exponential functions, symmetric with respect to $y_{0}$. Qualitatively speaking, the long- $\lambda$ modes have lost functional "plasticity" compared with solutions corresponding to $\lambda \backsim \Delta$.

From eq. 5 one can see that the matching conditions at $y=y_{0}$ are

$$
[\zeta]=0, \quad\left[\mathcal{H} \frac{d \zeta}{d y}\right]=0,
$$

where the bracket is a shorthand for $[Q]=$ $\lim _{\epsilon \rightarrow 0}\left[Q\left(y_{0}+\epsilon\right)-Q\left(y_{0}-\epsilon\right)\right]$. This is the basis of the TD model, which leads to the dispersion relation

$$
\mathcal{H}_{1}+\mathcal{H}_{2}=0
$$

where the suffixes 1 and 2 indicate the values of the physical quantities on either side of the discontinuity.

\section{The unstable spectra and the mode weight analogy}

The square of the absolute value of the Lagrangian displacement

$$
|\zeta|^{2}=\Re(\zeta)^{2}+\Im(\zeta)^{2}
$$

and the square of the absolute value of the derivative of $\zeta$ play an important role in the determination of the spectrum properties of the characteristic values $c=c_{r}+i c_{i}$. In fact, to discuss the spectral properties of the $\mathrm{KH}$ instability we may introduce a quantity analogous to a density distribution, which we may call the mode weight, defined by

$$
M(y) \equiv \rho_{0}(y)\left[\left|\frac{d \zeta}{d y}\right|^{2}+k^{2}|\zeta|^{2}\right] .
$$

In this conceptual analogy $M(y)$ is associated not only to the equilibrium density $\rho_{0}(y)$ but depends also on the amplitude and the localization of the perturbative mode via the bracket containing $|\zeta|^{2}$ and $|d \zeta / d y|^{2}$. The mode weight is reminiscent of techniques used in the Rayleigh-Ritz method for the study of the Sturm-Liouville spectral theory, although the characteristic value dealt here is not an eigenvalue problem of the latter family, and therefore some powerful classic properties do not hold.

Starting from equation 5, multiplying by $\zeta^{*}$ and integrating over $y$, we find after integration by parts the following condition

$$
\int \mathcal{H}\left[\left|\frac{d \zeta}{d y}\right|^{2}+k^{2}|\zeta|^{2}\right] d y=0
$$

We have assumed that $\zeta \rightarrow 0$ as $y \rightarrow \pm \infty$, which is certainly the case for configurations with two asymptotic regions with constant fields, separated by a velocity gradient region where all the fields change. In mathematical terms, the formula 15 is of the class of "energy integrals".

Separating the real and the imaginary part of equation 15 , it is easy to show that the real and imaginary part of the phase velocity, $c=c_{r}+i c_{i}$, for the unstable spectrum $c_{i} \equiv \Im(c)>0$, are given by the expressions indicated in the following. The real part of the phase velocity is equal to the speed of a fictitious center of mass

$$
c_{r}=\frac{\int V_{\kappa}(y) M(y) d y}{\int M(y) d y}, \quad c_{i} \neq 0,
$$

computed with the mode weight. Clearly, the weight distribution decides which value of the $V_{\kappa}(y)$ profile is being emphasized by a physically admissible perturbation, and becomes the phase velocity of the growing wave. The importance of equation 16 is that it shows that the phase velocity is restricted to the range $V_{\kappa m}<c_{r}<V_{\kappa M}$, where $V_{\kappa m}, V_{\kappa M}$ are the minimum and maximum values of $V_{\kappa}(y)$, respectively, and thus the excited wave is resonant with some plasma layer of the velocity gradient region. We must stress that this holds only when $c_{i} \neq 0$.

The growth rate of the instability, instead, is given by the difference of two positive terms. The first, the driving term of the instability, is the quadratic spread of the velocity projection $V_{\kappa}(y)$ with respect to the center of mass velocity. The second term represents the stabilizing influence of magnetic tensions, and is equal to the quadratic mean of the projected Alfvén velocity 


$$
c_{i}^{2}=\frac{\int\left(V_{\kappa}(y)-c_{r}\right)^{2} M(y) d y}{\int M(y) d y}-\frac{\int V_{A \kappa}(y)^{2} M(y) d y}{\int M(y) d y}, \quad c_{i}>0 .
$$

The formula reminds at a distance a similar Rayleigh quotient of the mathematical literature of spectral problems.

It is convenient, starting from the mode weight $M(y)$, to define also a normalized mode distribution

$$
f(y) \equiv \frac{M(y)}{\int M(y) d y},
$$

satisfying

$$
\int f(y) d y=1
$$

We can then rely on the analogy of $f(y)$ with probability functions, also positive definite, and normalized to unity. Thus, we may define the average of any physical quantity $Q(y)$ with respect to the mode distribution as,

$$
\langle Q\rangle \equiv \int Q(y) f(y) d y
$$

in the customary way averages are defined in the theory of probability.

The spectral theorem, embodied in equations 16 and 17 , can then be rewritten in the following form

$$
\begin{aligned}
c_{r} & =\left\langle V_{\kappa}\right\rangle, \\
c_{i}^{2} & =\left\langle\left(V_{\kappa}-\left\langle V_{\kappa}\right\rangle\right)^{2}\right\rangle-\left\langle V_{A \kappa}^{2}\right\rangle, \quad c_{i}>0,
\end{aligned}
$$

using the first and second order moments of the mode distribution $f(y)$. We noted already the meaning of the first equation, when the average $\left\langle V_{\kappa}\right\rangle$ has been determined by a mode distribution, the growth of the instability given by the second equation 21 depends on the quadratic deviation of $V_{\kappa}(y)$ with respect to the mean value, but is reduced by the average of $V_{A \kappa}^{2}$. The first term in $c_{i}^{2}$ relies on the presence of a region of velocity gradient for $V_{\kappa}(y)$ (i.e., vorticity, $\operatorname{rot}(\vec{V})$ ) because it is zero when $V_{\kappa}$ is a constant. On the other hand, the stabilizing term is due to the magnetic tensions that arise from the perturbation $\zeta(y)$, which modifies the curvature of the magnetic lines that are straight in equilibrium.

To favor the excitation of the instability, a perturbation should have a peak of $f(y)$ at a $y$ position that gives a significant spread to $\left(V_{\kappa}-\left\langle V_{\kappa}\right\rangle\right)^{2}$, while the same location and peak should produce only a small average $V_{A \kappa}^{2}$. It is evident that very strong magnetic fields may not permit the existence of such a perturbation. However, in general, because of the difference of the profile functions $V_{\kappa}(y)$ and $V_{A \kappa}(y)$, it may be possible to have $y$ intervals where the former dominates over the latter. In that case, when the mode is physically admissible, a peak of $f(y)$ in that specific interval may emphasize the quadratic spread of $V_{\kappa}-\left\langle V_{\kappa}\right\rangle$ over the average $V_{A \kappa}^{2}$, and produce the amplification of the perturbation.

As an example of application of the spectral eqs.21, we show how the dispersion relation for a TD can be derived from this theorem. The TD is defined by two sets of parameters, $V_{\kappa}^{1,2}, V_{A \kappa}^{1,2}$, and $\rho_{1,2}$ for regions $1, y>0$, and 2 , $y<0$, separated by an interface at $y=0$, where the physical quantities suffer jumps. The solution of eq.5 for this case, taking into account the continuity of $\zeta$ at $y=0$, is

$$
\begin{aligned}
& \zeta_{1}=A \exp (-k y), \quad y>0 \\
& \zeta_{2}=A \exp (k y), \quad y<0,
\end{aligned}
$$

so that the mode function $f(y)$ for this case is given by

$$
f(y)=\frac{\rho_{1}}{\rho_{1}+\rho_{2}} 2 k \exp (-2 k y), \quad y>0, f(y)=\frac{\rho_{2}}{\rho_{1}+\rho_{2}} 2 k \exp (2 k y), \quad y<0 .
$$

Averaging $V_{\kappa}$ with 23 we immediately obtain the phase velocity of the modes,

$$
c_{r}=\left\langle V_{\kappa}\right\rangle=\frac{\rho_{1} V_{\kappa}^{1}+\rho_{2} V_{\kappa}^{2}}{\rho_{1}+\rho_{2}}
$$

provided $c_{i} \neq 0$.Computing $c_{i}^{2}$ with eq.21 we find

$$
\begin{aligned}
c_{i}^{2} & =\frac{\rho_{1}}{\rho_{1}+\rho_{2}}\left(V_{\kappa}^{1}-\left\langle V_{\kappa}\right\rangle\right)^{2}+\frac{\rho_{2}}{\rho_{1}+\rho_{2}}\left(V_{\kappa}^{2}-\left\langle V_{\kappa}\right\rangle\right)^{2}-\frac{\left(B_{\kappa}^{1}\right)^{2}+\left(B_{\kappa}^{2}\right)^{2}}{4 \pi\left(\rho_{1}+\rho_{2}\right)}= \\
& =\frac{\rho_{1} \rho_{2}}{\left(\rho_{1}+\rho_{2}\right)^{2}}\left(V_{\kappa}^{1}-V_{\kappa}^{2}\right)^{2}-\frac{\left(B_{\kappa}^{1}\right)^{2}+\left(B_{\kappa}^{2}\right)^{2}}{4 \pi\left(\rho_{1}+\rho_{2}\right)},
\end{aligned}
$$


completing an alternative deduction of the well-known dispersion relation for a TD. The stability criterium 1 is a direct consequence of eq. 25 .

In section 5 we use the mode distribution function and the spectral theorem to explain the existence of the long wavelength boundary of the KH instability.

\section{A stability analysis solved in closed form}

In this model a sharp density drop and a variation of $B_{\kappa}$ occur over a distance $\delta$, much smaller than the thickness, $\Delta=2 d$, where the gradient of the velocity $V_{\kappa}$ occurs. When there is a wide difference between the two scales, $\delta \ll \Delta$, we can obtain an exact solution of eq. 5 assuming step functions for $\rho_{0}, B_{\kappa}$, and a linear velocity profile for $V_{\kappa}$ in the interval $-d \leq y \leq d$. The model ignores the field structure within $\delta$, which is possible when the wavelength of the perturbation is such that the condition $\delta \ll \lambda$ holds. Thus, this model represents the changes of $\rho_{0}$ and $B_{\kappa}$ with discontinuous jumps through an interface of zero thickness $(\delta \rightarrow 0)$ located at $y=-d$, where matching conditions like eq.11 apply.

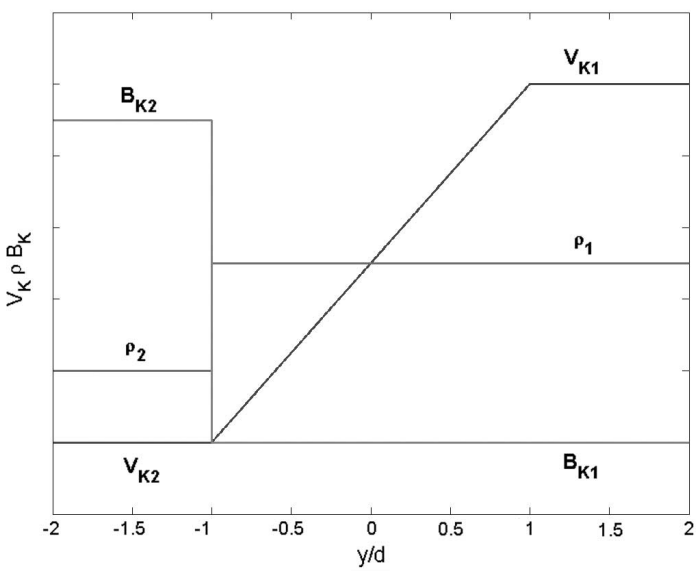

Figure 1. Schematic of the idealized model that can be solved in closed form. Field profiles $B_{\kappa}, V_{\kappa}$, and $\rho_{0}$ as functions of $y / d$, the vertical scale is arbitrary; $V_{\kappa}$ joins two intervals of constant values $V_{\kappa 1}, V_{\kappa 2}$ with a linear function in the interval $y / d=(-1,1)$; $B_{\kappa}$ and $\rho_{0}$ are constant functions with a discontinuous jump at $y / d=-1$; the values of $B_{\kappa 1}$, and $V_{\kappa 2}$ are zero (see text).

The schematic for this model is given in Fig. 1, which shows step functions for both $\rho_{0}$ and $B_{\kappa}$, while $V_{\kappa}$ is represented by a continuous, albeit broken profile. The latter has a linear transition of width $2 d$ between two constant velocity values, the one on the left chosen to be zero. In Fig. 1 the horizontal $y$ axis is normalized with $d$, but the vertical scale is only qualitative, and indicates the trend of the three quantities, $\rho_{0}, B_{\kappa}$, and $V_{\kappa}$. The reader should note that the configuration does not require $\overrightarrow{B_{0}}$ to be zero in the interval $-d \leq y<\infty$, but only that the magnetic field $\overrightarrow{B_{0}}$ is unidirectional there. We are considering modes with a $\vec{k}$-vector normal to the magnetic field $\left(\vec{k} \perp \overrightarrow{B_{0}}\right)$ in that region. At $y=-d$ a current sheet exists and the direction of $\overrightarrow{B_{0}}$ rotates sharply, so that $B_{\kappa} \neq 0$ when $-\infty<y<-d$, an interval in which the magnetic field becomes unidirectional again but with a different orientation.

The purpose of this model is only to prove with an exact solution, easy to handle and discuss, the thesis of the paper. It does not intend to represent realistic features for any particular application.

With the change of variable

$$
w=\sqrt{\mathcal{H}} \zeta
$$

eq. 5 is transformed into

$$
\frac{d^{2} w}{d y^{2}}-\left(k^{2}+\frac{1}{\sqrt{\mathcal{H}}} \frac{d^{2} \sqrt{\mathcal{H}}}{d y^{2}}\right) w=0 .
$$

We note that, since $B_{\kappa}=0$ in the range of $-d \leq y<\infty$, then $\mathcal{H}=\rho_{1}\left(c-V_{\kappa}(y)\right)^{2}$, where $\rho_{1}$ is constant there. Within the region of constant density and linear dependence of $V_{\kappa}$ with $y$ (constant $d V_{\kappa} / d y$ ) we see that

$$
\frac{d^{2} \sqrt{\mathcal{H}}}{d y^{2}}=0
$$

Then, the solution of eq. 5 for the slab $-d \leq y \leq d$ is given by

$$
\zeta=\frac{1}{\sqrt{\rho_{1}}\left(c-V_{\kappa}(y)\right)}\left[C_{1} \exp (-k y)+C_{2} \exp (k y)\right],
$$

where $C_{1,2}$ are constants, and

$$
V_{\kappa}(y)=\frac{1}{2} U_{1}\left(1+\frac{y}{d}\right),
$$

where we have written $U_{1}=V_{\kappa}(d)$ and set $V_{\kappa}(-d)=0$.

For the interval $1, d<y$, the solution can be written as

$$
\zeta=\frac{A_{1} \exp (-k y)}{\sqrt{\rho_{1}}\left(c-U_{1}\right)},
$$

whereas in the interval $2, y<-d$, the solution is given by

$$
\zeta=\frac{A_{2} \exp (k y)}{\sqrt{\rho_{2}} c},
$$

with constant density $\rho_{2}$ in that region

The dispersion relation is derived from two sets of matching conditions: one set is for $y=-d$,

$$
\left[\mathcal{H} \frac{d \zeta}{d y}\right]=0, \quad[\zeta]=0,
$$

where there are jumps of $\rho_{0}$ and $B_{\kappa}$, so that, $\mathcal{H}_{s}=\rho_{1} c^{2}$, for the value of $\mathcal{H}$ on the slab side, and $\mathcal{H}_{2}=\rho_{2} c^{2}-B_{2}^{2} / 4 \pi$ for the opposite side. The quantity $B_{2}=B_{\kappa}(-d)$ is the constant value of the magnetic field projection on $\vec{\kappa}$ in region 2 . The other boundary conditions are at $y=d$

$$
[\zeta]=0, \quad\left[\frac{d \zeta}{d y}\right]=0
$$


where all the fields are continuous (and $B_{\kappa}=0$ ). There are four coefficients, $A_{1,2}$ and $C_{1,2}$, in the solution and four boundary conditions, which are linear homogeneous equations for these coefficients. The compatibility condition for this set of equations requires a $4 \times 4$ determinant to be zero, which leads to an equation for $\omega$, as is customary in this type of problems.

The algebra is simple although laborious, omitting details the resulting polynomial in $\widehat{c}$ can be written as

$$
\begin{aligned}
& 2 \alpha\left(1+r_{d}\right) \widehat{c}^{3}+\left[\alpha(1-2 \alpha)\left(1+r_{d}\right)-2 \alpha+\alpha \exp (-2 \alpha)\left(1-r_{d}\right)\right] \widehat{c}^{2} \\
& -\left[1-2 \alpha+2 \alpha P^{2}-\exp (-2 \alpha)\right] \widehat{c}-(1-2 \alpha-\exp (-2 \alpha)) \alpha P^{2} \\
= & 0
\end{aligned}
$$

which gives the dispersion relation for the model with a linear velocity slab and discontinuities in density and magnetic field. The meaning of the symbols in eq. 34 is the following

$$
\alpha=2 k d, \quad \widehat{c}=\frac{c}{U_{1}}=\frac{\omega}{k U_{1}}, \quad r_{d}=\frac{\rho_{2}}{\rho_{1}}, \quad P^{2}=\frac{B_{2}^{2}}{4 \pi \rho_{1} U_{1}^{2}}=\frac{1}{M_{A}^{2}} .
$$

The nondimensional parameter $P$ is the inverse of a particular type of Alfvénic Mach number, $M_{A}$, defined with the velocity projection $U_{1}$ and density $\rho_{1}$ of region 1 , and the magnetic field projection of region 2 .

In eq.34 $\lambda$ and $\Delta=2 d$ may take arbitrary values. The limit $d \rightarrow 0$ when $\alpha \rightarrow 0$, requires the expansion of eq.34 in a power series of $\alpha$. The lower orders $\alpha^{0}, \alpha^{1}$, are satisfied identically. The lowest significant order is $\alpha^{2}$, and leads to

$$
\left(1+r_{d}\right) \widehat{c}^{2}-2 \widehat{c}+1-P^{2}=0
$$

which is the same polynomial that can be derived from eq. 12 when $\mathcal{H}_{1}=\rho_{1}\left(c-U_{1}\right)^{2}$ and $\mathcal{H}_{2}=\rho_{2} c^{2}-B_{2}^{2} / 4 \pi$. In this limit the slab $-d \leq y \leq d$ reduces to a TD, so that both eq. 25 and the roots of eq. 36 in this case give

$$
\widehat{c}=\frac{\omega}{k U_{1}}=\frac{1}{1+r_{d}}\left[1 \pm \sqrt{\left(1+r_{d}\right) P^{2}-r_{d}}\right] .
$$

Thus, the condition for KH stability in the long wavelength limit of the model of Fig. 1 is

$$
\frac{1}{M_{A}^{2}} \geq P_{c}^{2} \equiv \frac{r_{d}}{\left(1+r_{d}\right)}
$$

which corresponds to the condition

$$
V_{A 2}^{2}=\frac{B_{2}^{2}}{4 \pi \rho_{2}} \geq \frac{U_{1}^{2}}{\left(1+r_{d}\right)} .
$$

Next, we assume that condition 38 is satisfied, and holding the same parameters for regions 1 and 2, i.e., for equal $M_{A}$ and $r_{d}$, we analyze the dispersion relation for finite $\Delta=2 d$ as a function of $\alpha=2 k d$. Thus, we proceed to compute numerically the roots of eq.34, which are shown in Fig. 2. As an example, for a density ratio $r_{d}=\rho_{2} / \rho_{1}=0.1$, and $P=0.43$ that corresponds to $M_{A}=2.3, P$ is about 1.44 times larger than the critical stability value for the corresponding TD, $P_{c}=0.3015$, so that condition 1 is satisfied.
Nevertheless, as we can see from Fig. 2 the system is unstable with a maximum growth rate $\gamma_{m} \approx 0.16 U_{1} / \Delta$ at about $k d=0.7$. In the range $k d \lesssim 0.22$, which is equivalent to $\lambda>\lambda_{l} \gtrsim 14.3 \times \Delta$, all the modes (with the same orientation $\vec{\kappa}$ ) are stable, so that the result is consistent with the TD criterium that predicts stability at very long $\lambda \mathrm{s}$.

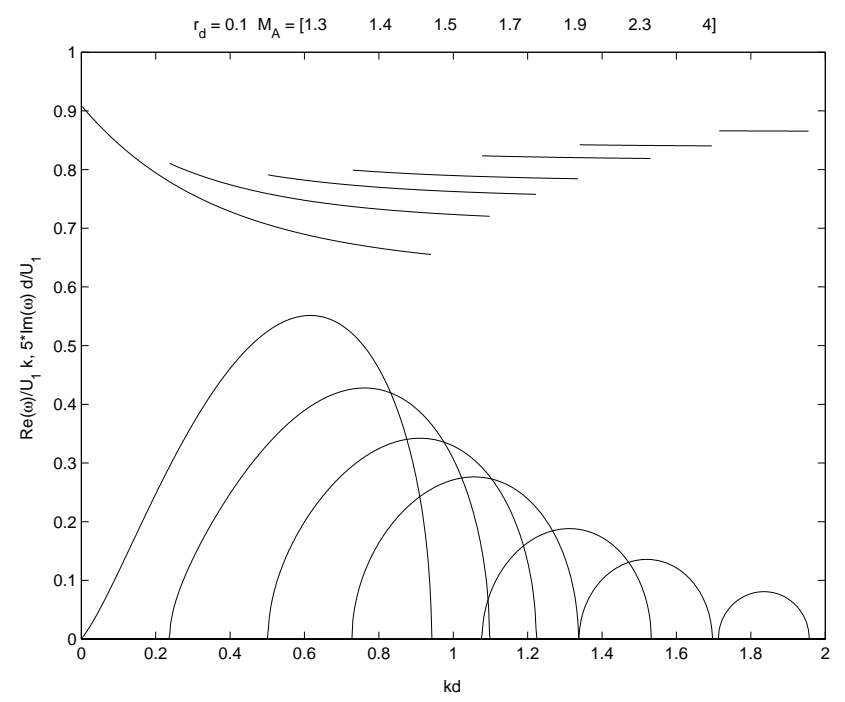

Figure 2. $\Re(\omega) / k U_{1}$, upper line segments, and $5 \times \Im(\omega) d / U_{1}$, bottom arcs, as functions of $k d$ for $r_{d}=0.1$ from roots of the dispersion relation eq.34. The different lines correspond to $M_{A}=(1.3$, $1.4,1.5,1.7,1.9,2.3,4)$ starting from the right with $M_{A}=1.3$ and increasing to the left up to $M_{A}=4$.

In Fig. 2, the growth rate is normalized with $U_{1} / d$, and the plot shows $5 \times \Im\left(\omega d / U_{1}\right)$ versus $k d$, so that the real part of $\omega$ may be shown in the same diagram. On the same vertical scale, the normalized phase velocity $\Re(\omega) / k U_{1}$ is given 
too. The figure gives the roots of (34) for $r_{d}=0.1$, and seven values of $M_{A}=(1.3,1.4,1.5,1.7,1.9,2.3,4)$. The upper line segments of the plot give the phase velocity of the modes for which $5 \times \gamma d / U_{1}$ is represented by the curves in the lower part. The maximum growth rate of these curves increases steadily with increasing $M_{A}$. We may also note that the curves for $5 \times \Im\left(\omega d / U_{1}\right)$ are shifted from right to left as $M_{A}$ increases, while the unstable $k d$ intervals also grow systematically. Finally, between $M_{A}=2.3$ and $M_{A}=4$, more precisely for $M_{A}^{c}=3.317$, the long wavelength boundary disappears, in coincidence with the fact that the TD becomes unstable. For $M_{A}>M_{A}^{c}$ both the TD and the model with a finite thickness are unstable, however the growth rates computed with eq. 25 are reasonable estimates only when $\lambda \gg \Delta$, otherwise they are unreliable.

Figure 2 also shows the presence of the short $\lambda$ boundary, which is a well-known characteristic of the KH instability, and occurs also in ordinary fluids (no magnetic fields). For instance, the plot shows for $M_{A}=2.3$ a limit at $k d \approx 1.1$, i.e., for $\lambda_{s} \approx 2.9 \Delta$, so that the interval $0<\lambda<\lambda_{s}$ is also stable.

The phase velocity of the unstable modes is nearly constant at the smaller values of $M_{A}$, but shows more variation when $M_{A}$ is large, with $\Re(\omega) / k$ as a decreasing function of $k$, so that the group velocity is smaller than the phase velocity.

Of course, the results and comments of this section are for a particular choice of $\vec{\kappa}$, the study of a different orientation of the $\vec{k}$-vector would need new computations for different functions $V_{\kappa}$ and $B_{\kappa}$, as already noted.

\section{The equivalent weight of unstable modes}

We show now why it may be possible that the modes are stable at long $\lambda \mathrm{s}$, and yet be unstable at smaller wavelengths. Since the modes are known from the solutions of section 4 we can compute $\zeta$ in closed form. As an example, Fig. 3 shows the shape of $\Re(\zeta)$, the real part of the $y$-component of the Lagrangian displacement (see eq.5) for a particular unstable mode of the model of section 4 , corresponding to $r_{d}=0.1, M_{A}=2.3$, and $k d=0.6$, with $\gamma=0.156 U_{1} / \Delta$. For the same $\vec{\kappa}$ orientation, modes with very long $\lambda \mathrm{s}$ are stable since $M_{A}<M_{A}^{c}=3.317$. For $k d=0.6$ we note that $\Re(\zeta)$ has a neat peak near $y \sim 0.5 d$ in a range of $y$ where $B_{\kappa}=0$. At $y=0.5 d$ the value of $V_{\kappa}$ is 0.75 , while the phase velocity, equal to $\left\langle V_{\kappa}>\right.$ according to equation 21 , is $c_{r}=0.7$. The peak of $\Re(\zeta)$ does not extend (with significant amplitude) into the $y$ interval $(y<-d)$ where the magnetic tension generated by $B_{\kappa} \neq 0$ provides a stabilizing effect. The imaginary part, $\Im(\zeta)$ (not shown) has similar features.

Figure 4 gives the corresponding mode distribution function $f(y)$ computed from eq.14. We can see that the normalized $M(y)$ is strongly localized in a $y$ interval where $\left(V_{\kappa}-<V_{\kappa}>\right)^{2}$ is significantly different from zero, while $V_{A \kappa}^{2}=0$, and that $M(y)$ is very small in the range of $y$ where $V_{A \kappa}^{2}$ is different from zero. The peak of $M(y)$ in
Fig. 4 appears much enhanced with respect to that of $\Re(\zeta)$ in Fig. 3. This is not only due to $|\zeta|^{2}$, but mainly to the contribution of $|d \zeta / d y|^{2}$.

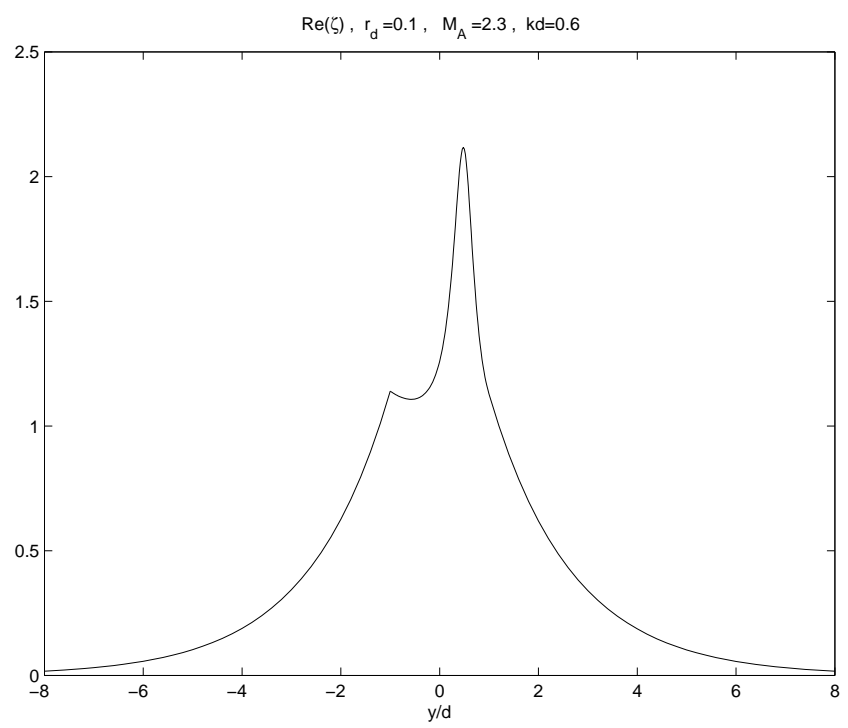

Figure 3. $\Re(\zeta)$ as a function of $y / d$ for the unstable mode of the model of section 4 with $r_{d}=0.1, M_{A}=2.3$, and $k d=0.6$. Note that the maximum value occurs in a $y / d$ interval where $B_{\kappa}=B_{\kappa 1}=0$ (see Fig. 1).

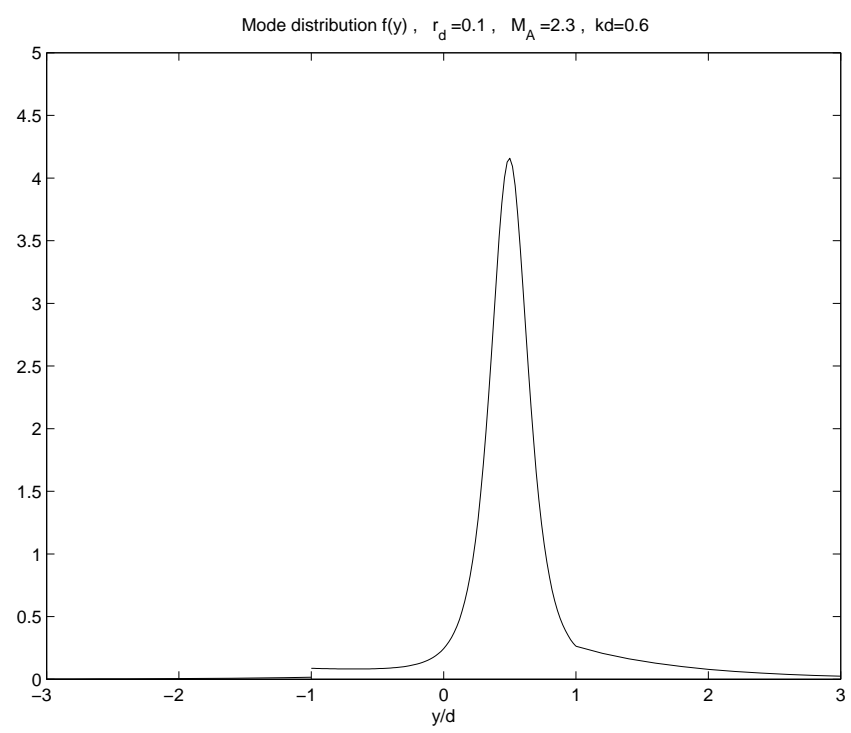

Figure 4. Example of a mode distribution $f(y)$ versus $y / d$ for the same unstable mode of Fig.3 $\left(r_{d}=0.1, M_{A}=2.3, k d=0.6\right)$. The peak is strongly localized in the interval $y / d=(0,1)$ where $V_{A \kappa}=0$ (see text).

The possibility of such a strong localization of the mode distribution $f(y)$ for moderate $\lambda$ s allows the inequality $\left.\left\langle\left(V_{\kappa}-\left\langle V_{\kappa}\right\rangle\right)^{2}\right\rangle\right\rangle\left\langle V_{A \kappa}^{2}\right\rangle$ to be satisfied, and hence the development of the instability, in agreement with eq.21. 
Conversely, modes with very long wavelengths, such that $\lambda \gg \Delta$, are broadly spread over and much beyond the interval $\Delta=2 d$. In fact, at these $\lambda s$, the solution $\zeta$ becomes necessarily close to exponential functions that decay symmetrically on both sides of the transition, as can be seen from equation 22. Flexibility in localization is not possible at these long $\lambda \mathrm{s}$, the mode distribution has a fixed shape, a broad symmetric peak about $y=0$, where the velocity gradient region is concentrated. This kind of mode distribution cannot avoid to give a significant contribution to the average of $V_{A \kappa}^{2}$, arising from intervals where $V_{A \kappa}^{2} \neq 0$. Therefore, at long wavelengths the inequality may be reversed, $\left\langle\left(V_{\kappa}-\left\langle V_{\kappa}\right\rangle\right)^{2}\right\rangle\left\langle\left\langle V_{A \kappa}^{2}\right\rangle\right.$, leading to stability as in the case of the TD criterium.

\section{Hyperbolic tangent profiles and the case of uniform magnetic field.}

In this section we briefly comment on results of numerical integrations of eq.5 using continuous smooth profiles for all the fields, i.e., with finite values for the length scales $\Delta$ and $\delta$ defined in section 4. On one hand, the calculations show the significance of the idealized model of section 4 , ascertaining that the existence of a long wavelength stability boundary is confirmed working with smooth field profiles. On the other hand, it is found that a configuration without magnetic shear (uniform magnetic field) does not have the long $\lambda$ stability boundary for modes with $B_{\kappa} \neq 0$.

We have considered fields represented by hyperbolic tangent functions, with a length scale, $\Delta$, for $V_{\kappa}(y)$, and another scale, $\delta$, for both $B_{\kappa}$ and $\rho$. As an alternative, we examine also another configuration in which $B_{\kappa}=B_{0}$ is a constant, $\rho=\rho_{0}$ also remains constant, and only $V_{\kappa}$ varies as an hyperbolic tangent function. The equilibrium fields for both configurations are given by

$$
\begin{aligned}
V_{\kappa} & =\frac{U_{1}}{2}[1+\tanh (Y)] \\
B_{\kappa} & =\left\{\begin{array}{l}
\frac{B_{2}}{2}\left[1-\tanh \left(\frac{\Delta}{\delta}(Y+1)\right)\right] \\
B_{0}
\end{array}\right. \\
\rho & =\left\{\begin{array}{c}
\frac{1}{2}\left(1+r_{d}\right)+\frac{1}{2}\left(1-r_{d}\right) \tanh \left(\frac{\Delta}{\delta}(Y+1)\right) \\
\rho_{0}
\end{array}\right.
\end{aligned}
$$

where $Y=y / d$. It is worth noting that these steady state field profiles, as well as those of the analytic model (Fig. 1) of a previous section, are exact solutions of the steady state equations of ideal magnetohydrodynamics, eqs. 2. The hyperbolic tangent function was chosen only as a simple smooth function, continuous together with all its derivatives, that joins two intervals of constant value.

The numerical work was performed with a method of finite differences, applied to a set of first order differential equations equivalent to the second order eq.5. For the numerical computation with the two-scale model we have taken $\delta / \Delta=0.2$. The configuration with hyperbolic tangents for all the fields was intended to be close to the idealized example of Fig. 1.
We omit figures here for brevity, and report only that the trends revealed by the model of section 4 are qualitatively confirmed by the numerical computations. The hyperbolic tangent model also has a critical value $\lambda_{l}$, such that modes with $\lambda>\lambda_{l}$ are stable, corresponding to a cutoff of growth rates at small values of $k d$, similar to that shown in Fig. 2 for the idealized model. The maximum growth rate decreases with diminishing $M_{A}$ as expected, although with values somewhat smaller than those of Fig. 2. Also, the shift of the unstable intervals towards larger values of $k d$ for decreasing values of $M_{A}$ is much reduced in the case of the hyperbolic tangent model. The interested reader may visually compare the results of the hyperbolic tangent profiles with those of the model of section 4 in Gratton et al. [6] where the matter is discussed.

Here, we focus on the results for the alternative configuration with uniform magnetic field. Of course, in this case we may have flute modes with $\vec{k} \perp \vec{B}$ : these are always unstable, except at small $\lambda \mathrm{s}$, a range in which the velocity gradient operates as a stabilizing agent reversing its action as driver of the instability typical of longer wavelengths (see Gratton et al. [9]). Let us consider, instead, modes with $B_{\kappa}=B_{0} \neq 0$, for all $y$. A simple analytic solution for the linear velocity profile, like that of section 4 , is not possible in this case in spite of the simplicity of the magnetic field profile, and a numerical treatment is required.

We solve the stability problem for the second option of eqs. 41 . Fig. 5 gives the normalized growth rate $\Im(\omega) d / U_{1}$ as a function of $k d$ for a set of sixteen values of $M_{A}=$ $\sqrt{4 \pi \rho_{0}} U_{1} / B_{0}$, ranging from 8.2 down to 2.2 with steps of 0.375 . The stabilizing effect of the increasing magnetic field is evident, with the diminution of the growth rates and the shrinking of the unstable interval. More interestingly, Fig. 5 also clearly shows that in this case a long- $\lambda$ stability boundary does not appear.

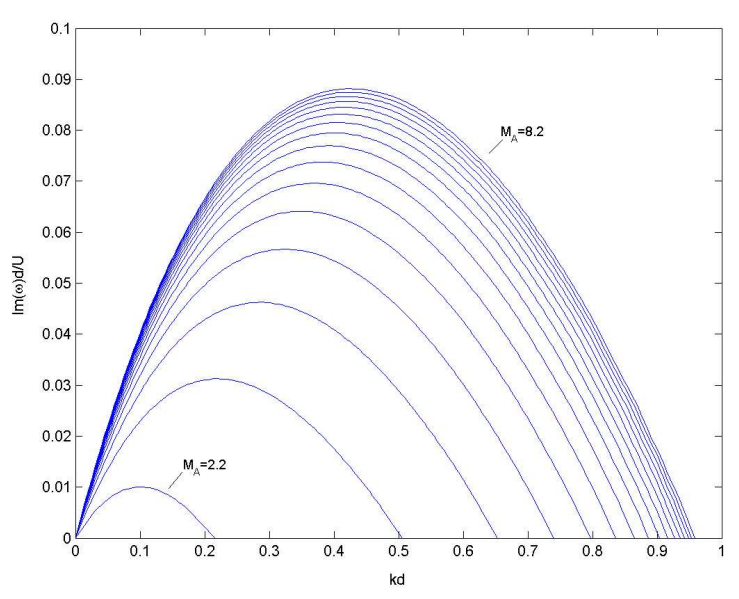

Figure 5. Normalized growth rate $\Im(\omega) d / U_{1}$ as a function of $k d$ for a $\mathrm{KH}$ configuration with uniform magnetic field and $B_{\kappa} \neq 0$ (see text). The lines correspond to sixteen values of $M_{A}$, from 8.2 down to 2.2 with steps of 0.375 . The plot shows that for this configuration without magnetic shear the lower $k d$ stability boundary (long $\lambda_{l}$ limit) is missing. 
Although the cause of the stabilization at long $\lambda \mathrm{s}$ is always the magnetic tension embodied in $B_{\kappa}^{2} \neq 0$, nevertheless the possibility of finding unstable modes at moderate $\lambda \mathrm{s}$ that become stable at long $\lambda \mathrm{s}$, is due to the presence of magnetic shear. This is because in the presence of magnetic shear there is a chance that modes may be localized in $y$ intervals of small or zero $B_{\kappa}^{2}$, as we have discussed in sections 4 and 5 .

\section{Stability Diagrams}

The flow with a constant magnetic field (second alternative in eqs.41) has a stability diagram shown in Fig. 6. The line gives the critical value of $k d$ that separates stable from unstable modes (for a fixed orientation $\vec{\kappa}$ ) as a function of $M_{A}$. For strong magnetic fields, $M_{A}<2$, the modes are stable for all wavelengths. When $M_{A}$ is larger than 2 but remains close to this value, $M_{A} \gtrsim 2$, the $k d$ interval of instability is small and the upper $k d \vec{d}$ limit corresponds to rather long wavelengths. However, the instability range starts always at $k d=0$. As $M_{A}$ increases significantly above 2 the stability boundary tends to the value $k d=1$ and clearly there is only a short $\lambda_{s}$ stability limit. A long- $\lambda$ stability boundary does not exist for this configuration.

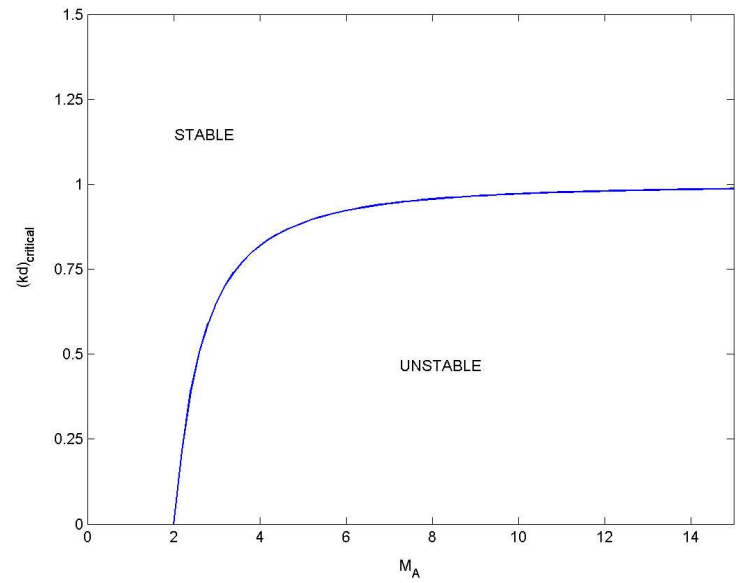

Figure 6. Stability diagram showing the critical $k d$ that separates stable from unstable regions as a function of $M_{A}$ for the same model of Fig. 5.

The stability diagrams for the model of section 4 , obtained from the dispersion relation 34, are shown in Figs. 7 and 8 and are very different from the diagram of Fig. 6. In Fig. 7 we present the stability diagram for a density ratio $r_{d}=0.1$ in a $k d$ vs. $M_{A}$ plane. The white area corresponds to stable modes, while the shaded regions are unstable. The curves that separate different gray areas correspond to contours of constant $\Im(\omega) d / U_{1}$. The critical $k d$ line is for $\Im(\omega) d / U_{1}=0$, and it encloses three contour lines of increasing growth rates, with $\Im(\omega) d / U_{1}=(0.03,0.06,0.09)$. In Fig. 7 there are two stability limits $k d$ for each $M_{A}$ value in the range $1.2 \lesssim M_{A} \lesssim 3.3$. These correspond to the short $\lambda_{s}$ and long $\lambda_{l}$ boundaries mentioned in the introduction. For $M_{A}>3.3$ only $\lambda_{s}$ exists and the instability range starts at $k d=0$. When $M_{A}<1.2$ the magnetic field is sufficiently strong to stabilize the configuration for all $\lambda \mathrm{s}$.

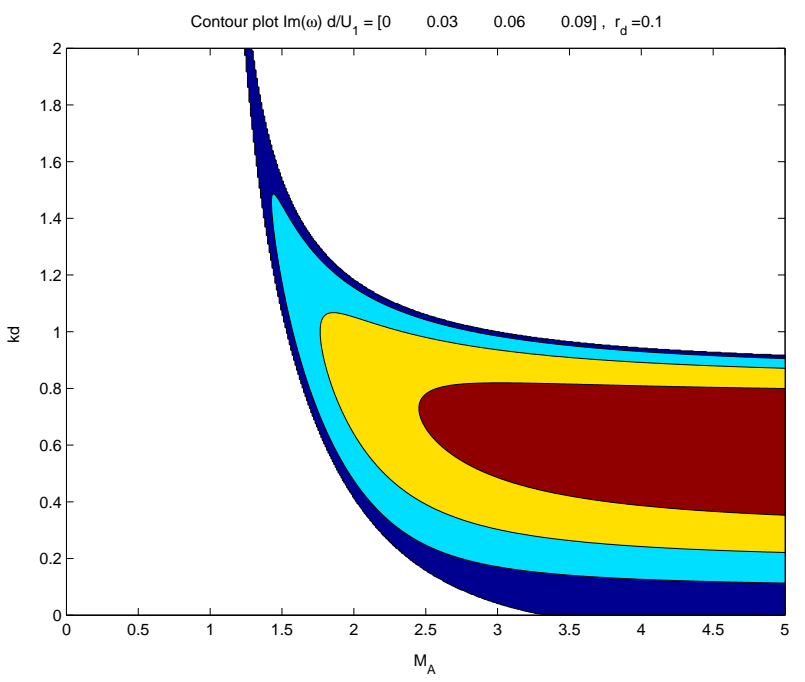

Figure 7. Stability diagram $k d$ versus $M_{A}$ for the model of section 4 with $r_{d}=0.1$. The white area is stable. Four contour lines for $\Im(\omega) d / U_{1}=(0,0.03,0.06,0.09)$ that separate shaded regions are also shown. In the interval $1.2 \lesssim M_{A} \lesssim 3.3$ two $k d$ critical values occur that correspond to the short $\lambda_{s}$ and long $\lambda_{l}$ stability boundaries.

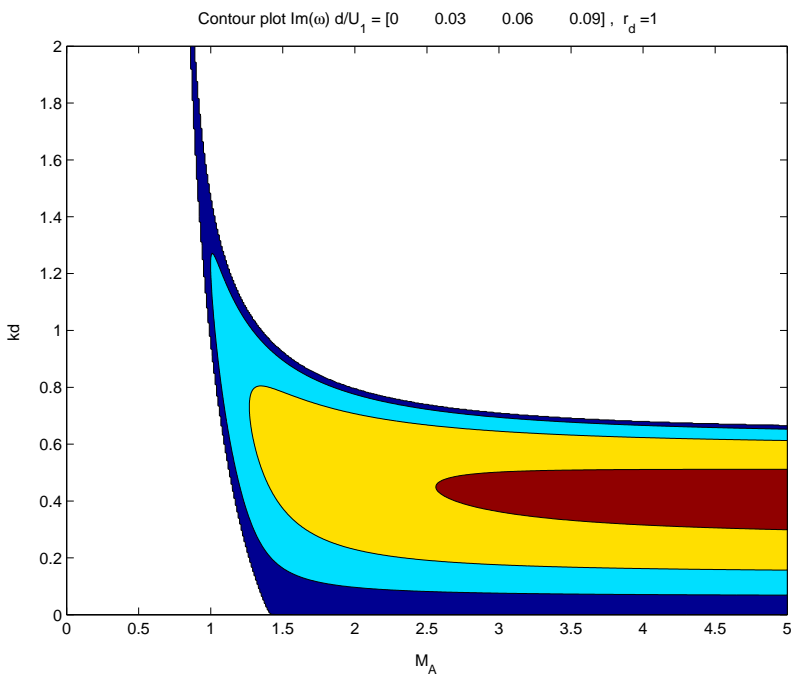

Figure 8 . Stability diagram $k d$ versus $M_{A}$ for the model of section 4 with $r_{d}=1.0$ with the same format of Fig. 7 . The plot illustrates the influence of the density ratio on the instability.

In Fig. 8 a stability diagram is given for $r_{d}=1$ with the same format of Fig. 7. The shape of the contour lines change with the density ratio. The unstable area in Fig. 7 looks like the bow profile of a boat. In Fig. 8 the bow profile has straightened up. The range of $M_{A}$ for which the unstable interval for $r_{d}=1$ has both short $\lambda_{s}$ and long $\lambda_{l}$ limits is much smaller than the one corresponding to $r_{d}=0.1$. 


\section{Conclusions}

We have shown with a theoretical example that a stable tangential discontinuity may turn out to be unstable when examined at short wavelengths, corresponding to modes that can explore the finite width structure of the interface. Although the configuration is not intended to be realistic, the example has the advantage that it can be solved in closed form and analyzed in detail. Numerical computation of the instability with more realistic hyperbolic tangent profiles, for the same parameters of the idealized model, confirms the results of the analytic study. This is only briefly stated here, but it is discussed in detail in Gratton et al. [6].

The fact that there are cases in which a long $\lambda_{l}$ stability boundary for $\mathrm{KH}$ exists, besides the well-known short $\lambda_{s}$ stability limit, as far as we know has not been noted in the literature. The possibility of the existence of a long $\lambda$ stability range relies on the presence of shear in the magnetic field configuration. It is absent for both flute and non flute modes of a unidirectional magnetic field, or in ordinary non conducting fluids. The well-known short wavelength stability interval of $\mathrm{KH}$, instead, is always present and occurs even in the absence of magnetic fields. We have explained the possibility of the existence of the long wavelength limit in MHD parallel flows, by means of a spectral theorem that allows a discussion of the physical effects operating on the instability, and their enhancement or reduction due to the possibility of strong localization of the modes at intermediate $\lambda \mathrm{s}$. We have shown also that this possibility is a MHD property, facilitated by configurations with magnetic shear, which is absent when the magnetic field is uniform.

The KH instability that amplifies surface waves, produces billows and vortices, and finally cascades into turbulence (e.g., Belmont and Chanteur [3]). These processes lead to diffusion and broadening of the velocity gradient, so that the thickness of the transition region increases (see, for instance, Miura [10]). The KH excitation, therefore, is important for the transport of momentum into the terrestrial magnetosphere, particularly during periods of northward orientation of the IMF (Farrugia et al.[5]). In relation to data analysis of spacecraft crossings, the reader should realize that the TD condition 1 is not sufficient to asses the KH stability of the magnetopause. To determine the theoretical stability of a $\mathrm{KH}$ configuration the finite thickness structure of the interface must be taken into account. On the other hand, when the TD is unstable, a more precise analysis will also confirm the instability. In that case, however, one should remember that the TD equation grossly overestimates the growth rate for $\lambda \sim \Delta$ and completely ignores the stabilization at short $\lambda \mathrm{s}$. Readers interested to see cases of practical application of the long stability limit to the study of the stability of the magnetopause are referred to Gratton et al. [6] where the subject is treated in detail.

The theory presented here is limited to subsonic flows. An extension of the stability diagrams for supersonic flows requires the inclusion of compressibility effects in the perturbative equations and remains a subject for future studies.

\section{Acknowledgments}

Research funded by the Argentinean grants UBACyT X059, X291, and CONICET PIP 2013. Also partially supported by USA NASA grants NAG5-13116, NAG5-11803. LB was a postdoc fellow of Fundación Bunge y Born, Buenos Aires, Argentina, during part of the work.

\section{References}

[1] S. Chandrasekhar, Hydrodynamic and hydromagnetic stability, Oxford University Press, New York, 1961.

[2] P.G. Drazin, and W. H. Reid, Hydrodynamic stability, Cambridge University Press, Cambridge, 1981.

[3] G. Belmont, and G. Chanteur, Physica Scripta, 124, 124 (1989).

[4] P. Huerre, and M. Rossi, Hydrodynamic instabilities in open flows, in Hydrodynamics and Non Linear Instabilities, C. Godreche and P. Manneville editors, Cambridge University Press, Cambridge, 1998.

[5] C.J. Farrugia, F. T. Gratton, and R. B. Torbert, Space Science Rev. 95, 443 (2001).

[6] F.T. Gratton, L. Bender, C. J. Farrugia, and G. Gnavi, J. Geophys. Res. 109, A04211 (2004).

[7] C.J. Farrugia, F. T. Gratton, L. Bender, H. K. Biernat, N. V. Erkaev, V. Denisenko, R. B. Torbert, and J. M. Quinn, J. Geophys. Res. 103, 6703 (1998).

[8] J. Contin, F. T. Gratton, and C. J. Farrugia, J. Geophys. Res. 108, 1227 (2003).

[9] F.T. Gratton, G. Gnavi, C. J. Farrugia, and L. Bender, J. Planet. \& Space Sci. 51, 769 (2003).

[10] A. Miura, J. Geophys. Res. 89, 801 (1984). 